\title{
Polymethacrylic acid grafted psyllium (Psy-g-PMA): a novel material for waste water treatment
}

\author{
Ranvijay Kumar • Kaushlendra Sharma • \\ K. P. Tiwary - Gautam Sen
}

Received: 2 February 2012/Accepted: 7 January 2013/Published online: 20 January 2013

(C) The Author(s) 2013. This article is published with open access at Springerlink.com

\begin{abstract}
Polymethacrylic acid grafted psyllium (Psy-gPMA) was synthesized by microwave assisted method, which involves a microwave irradiation in synergism with silver sulfate as a free radical initiator to initiate grafting reaction. Psy- $g$-PMA grades have been synthesized and characterized on structural basis (elemental analysis, FTIR spectroscopy, intrinsic viscosity study) as well as morphological and thermal studies, taking psyllium as reference. The effects of reaction time, amount of monomer and silver sulfate (free radical initiator) on grafting of PMA on psyllium backbone have been studied. It is observed that all the grades of Psy- $g$-PMA have higher intrinsic viscosities than that of psyllium. The best synthesized grade was Psy$g$-PMA having intrinsic viscosity of 6.93 and $58 \%$ grafting of PMA on the main polymer backbone. Further Psy- $g$ PMA applications as flocculants for waste water treatment have been investigated. Psy- $g$-PMA resulted in higher decrease in the flocculation parameters such as total dissolved solid or total solids compared to psyllium. Hence the result shows the possible application of grafted psyllium in wastewater treatment.
\end{abstract}

R. Kumar · K. Sharma $(\bowtie)$

Department of Applied Chemistry, Birla Institute of Technology, Patna 800014, Bihar, India e-mail: kkaushalbit@gmail.com

R. Kumar

e-mail: ranvijay143@gmail.com

K. P. Tiwary

Department of Applied Physics, Birla Institute

of Technology, Patna 800014, Bihar, India

G. Sen

Department of Applied Chemistry, Birla Institute of Technology, Mesra Ranchi 835215, India
Keywords Psyllium · Polymethacrylic Acid · Silver sulfate $\cdot$ Flocculation

\section{Introduction}

Improved living standard and increasing population needs recycling of wastewater to meet the demands of water. Flocculation is an effective way of treating wastewater as it removes the colloidal particles (as flock) and other contaminants that gets adsorbed in them. Natural polymers such as starch, sodium alginate, amylopectin, guargum, xanthan gum, kendu gum, chitosan, okra mucilage, and psyllium mucilage find extensive application as flocculants (Bolto et al. 1996; Singh et al. 2002; Khalil and Farag 1998; Samantaroy et al. 1997; Udaybhaskar et al. 1990; Agarwal et al. 2001; Mishra et al. 2002a). Psyllium is an anionic polysaccharide of $\mathrm{L}$-arabinose, $\mathrm{D}$-xylose and D-galactoronic acid.

Modifications of natural polymers to improve their chemical and physical properties are necessary to tailor the materials as per the requirements. Though natural polymers are cheap but they have shear instability, uncontrolled biodegradability, and varying efficiency. On the other hand, synthetic polymers are better treatment agents but are non-biodegradable and expensive. Hence, the interest in acrylamide grafted natural polymer has increased over the years owing to their multi-faceted application in many domains. Singh et al. (2000) reported a large number of graft copolymers of acrylamide (AM) with polysaccharides such as guargum, xanthan gum, sodium alginate, carboxylic methyl cellulose and starch using ceric ion $/ \mathrm{HNO}_{3}$ acid as redox initiator. Ceric ammonium nitrate (CAN) induced grafting of AM with psyllium is directed towards understanding the influence of concentrations of AM and CAN 
in the reaction mixture, on percentage grafting by preparing different samples (Mishra et al. 2002b). Polyacrylonitrile (PAN) grafted natural polymers on chitosan has been reported for removal of heavy metal ion (Kang et al. 1999). The use of Psy-g-PAN as a treatment agent for suspended solids (SS) and total dissolved solids (TDS) removal from textile waste and tannery wastewater has been reported (Rajani et al. 2002; Mishra et al. 2004).

The main problem in case of graft copolymers is the lack of available commercial methods for synthesis. One of the methods applied for grafted copolymers (polysaccharides) involve use of chemical free radical initiator (conventional method) in which free radical initiator (e.g. ceric ammonium nitrate or CAN, potassium per sulfate, etc.) generates free radical sites on polysaccharides molecule where monomers are added. The other methods to synthesize grafted copolymer include gamma, X- ray, and UV technique. However, these radiations are not suitable due to low productivity, high energy irradiation, and low penetration power respectively (da Silva et al. 2007; Mostafa 1995; Gupta and Sahoo 2001; Sen et al. 2009; Sen and Pal 2009; Wang et al. 2008; Vahdat et al. 2007). One of the best method of graft copolymer synthesis is microwave irradiation either by microwave initiated method in which microwave irradiation self generates free radical sites on polysaccharides backbone (Sen et al. 2009) or microwave assisted method which includes free radical initiator and microwave irradiation both to create free radical sites on the backbone of polysaccharides on which monomer molecules attached (Mishra et al. 2011). Microwave assisted method is rapid, highly reliable, and reproducible and yields high quality products compared to that of conventional method. Polyacrylamide grafted starch (St- $g$-PAM) by 'microwave assisted' method, in presence of ceric ammonium nitrate (CAN) as free radical initiator has been reported for waste water treatment (Mishra et al. 2011). Further silver sulfate was used to synthesize sulfonated styrene/hydroxyethyl acrylatate/lauryl methacrylate terpolymeric cationic-exchange membrane in nitrogen atmosphere as an initiator (Shin et al. 2011).

In present study, an attempt has been made to synthesize polymethacrylic acid grafted psyllium (Psy-g-PMA) by 'microwave assisted' method, in the presence of silver sulfate as a free radical initiator. The synthesis was done by varying reaction time, concentration of monomer and initiator. Synthesized materials have been characterized on structural basis (FTIR, elemental analysis, intrinsic viscosity), thermal analysis (thermogravimetric analysis and differential scanning calorimetry) and on morphological basis by scanning electron microscope (SEM). The exploration of Psy- $g$-PMA for treating waste water on the basis of flocculation has also been explored.

\section{Materials and methods (Experimental)}

\section{Materials}

Psyllium was purchased from "The Dholpur Sat Isabgol Factory, Gujrat, India (lot no. SF-1843-A3)". Silver sulfate was purchased from E. Merck (lot no. MA8M572292), Mumbai, India. Methacrylic acid was purchased from $\mathrm{CDH}$ (lot no. SC9S590099), Mumbai, India. Annular grade acetone was supplied by E. Merck, Germany (lot no. SC 1F610141), and kaolin extra pure was purchased from Loba chemie, India (lot no. 04260.00500), Jar test apparatus (flocculator) six jar set make Ikon, India. All the chemicals were used as received; without further purification. The details of the instrument used were "25 Liters" LG microwave oven model number: MC7688DP, 900 Watt, India. The waste water was collected from main sewage of Birla Institute of Technology, Patna, Bihar, India.

\section{Synthesis}

Microwave assisted synthesis of polymethacrylic acid grafted psyllium (Psy-g-PMA) was done using silver sulfate as free radical initiator.

In the typical process, $1 \mathrm{~g}$ of psyllium was dissolved in $100 \mathrm{ml}$ of distilled water and catalytic amount of $\mathrm{Ag}_{2} \mathrm{SO}_{4}$ was added in it. The mixtures were mixed well and then desired amount of methacrylic acid was added. The reaction mixture was then transferred into $1 \mathrm{~L}$ reaction vessel and it was subsequently placed on the turntable of a microwave oven. Now, microwave irradiation of 900 watt of power was introduced. Periodically, the microwave irradiation was paused just before boiling of the reaction mixture starts $\left(65^{\circ} \mathrm{C}\right)$ and was cooled by placing reaction vessel in ice cold water. This was done to prevent homopolymerization and vapour formation of methacrylic acid which is toxic/carcinogenic.

The grafting of this microwave assisted synthesized Psy$g$-PMA was calculated as:

$\%$ grafting $=\left[\mathrm{W}_{2}-\mathrm{W}_{1}\right] / \mathrm{W}_{1}$,

where, $\mathrm{W}_{1}=$ weight of psyllium, $\mathrm{W}_{2}=$ weight of grafted copolymer.

The optimized grade has been determined through its higher percentage of grafting and intrinsic viscosity which is proportional to molecular weight. The synthesis details and the designation of the sample have been tabulated in the Table 1. From Table 1, it is observed that $0.177 \mathrm{~mol}$ of PMA and $1.5 \mathrm{~g}$ of silver sulfate produce better grafting result at $3 \mathrm{~min}$ and $900 \mathrm{~W}$ powers. The mechanism of microwave assisted grafting has been depicted in Fig. 1. 
Table 1 Polymethacrylic acid grafted psyllium (Psy-g-PMA) grades synthesized copolymers

\begin{tabular}{|c|c|c|c|c|c|c|}
\hline Grade & $\begin{array}{l}\text { Wt of } \\
\text { psyllium } \\
(\mathrm{gm})\end{array}$ & $\begin{array}{l}\text { Wt of silver } \\
\text { sulfate }(\mathrm{gm})\end{array}$ & $\begin{array}{l}\text { Amount of } \\
\text { PMA } \\
\text { (in mol) }\end{array}$ & $\begin{array}{l}\text { Time of } \\
\text { irradiation } \\
\text { (up to gel } \\
\text { formation) (s) }\end{array}$ & $\begin{array}{l}\% \\
\text { Grafting }\end{array}$ & $\begin{array}{l}\text { Intrinsic } \\
\text { viscosity }(\mathrm{dl} / \mathrm{g})\end{array}$ \\
\hline $\begin{array}{l}\text { Psy- } g- \\
\text { PMA I }\end{array}$ & 1.0 & 0.8 & 0.177 & 180 & 41 & 3.57 \\
\hline $\begin{array}{l}\text { Psy-g- } \\
\text { PMA II }\end{array}$ & 1.0 & 1.0 & 0.177 & 180 & 47 & 6.19 \\
\hline $\begin{array}{l}\text { Psy- } g- \\
\text { PMA } \\
\text { III }\end{array}$ & 1.0 & 1.5 & 0.177 & 180 & 58 & 6.93 \\
\hline $\begin{array}{l}\text { Psy- } g- \\
\text { PMA } \\
\text { IV }\end{array}$ & 1.0 & 1.5 & 0.177 & 150 & 35 & 4.52 \\
\hline $\begin{array}{l}\text { Psy- } g \text { - } \\
\text { PMA V }\end{array}$ & 1.0 & 1.5 & 0.177 & 210 & 45 & 5.39 \\
\hline Psyllium & 1.0 & - & - & - & - & 2.55 \\
\hline
\end{tabular}

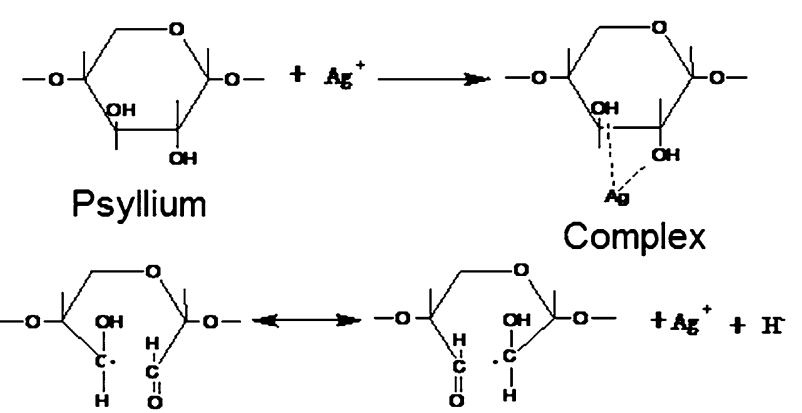

Free radical from psyllium ( $\left.\mathrm{R}^{\circ}\right)$

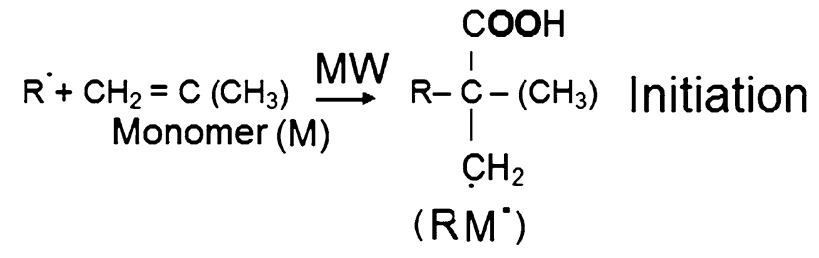

Propagation

Termination

\section{$M=$ Monomer \\ $\mathrm{MW}=$ Microwave $\mathrm{R}^{*}=$ Psyllium with free redical sites}

Fig. 1 Scheme for polymerization of PMA and grafting of PMA on psyllium backbone

The mechanism involves the $\mathrm{Ag}^{+}$generated free radical which participates in the formation of a chelating complex between the hydroxyl group of the psyllium and the oxidant (Fig. 1). The complex so formed disproportionate the forming of free radicals onto the polysaccharides (psyllium) backbone. The microwave radiation rotates the

methacrylic acid molecule leading to the elongation of its bonds. As the $\mathrm{C}-\mathrm{C}$ double bond electron cloud splits up into two localized clouds, the free radical sites created on the psyllium backbone by silver sulfate and on the methacrylic acid by microwave irradiation interacts through usual free radical reaction mechanism to yield the graft copolymer.

Purification of the graft copolymer by solvent extraction method

Any occluded polymethacrylic acid (PMA) formed by competing homopolymer formation reaction was removed from the grafted copolymers synthesized as above, by solvent extraction using a mixture of formamide and acetic acid (1:1 by volume).

\section{Characterization techniques}

The FTIR spectrums of Plantago psyllium mucilage and that of Psy- $g$-PMA were recorded in solid state, by $\mathrm{KBr}$ pellet method using a FTIR spectrophotometer (Model IRPrestige 21, Shimadzu Corporation, Japan) between 400 and $4,000 \mathrm{~cm}^{-1}$. The elemental analysis was undertaken with an elemental analyzer (Make-M/s Elemental Analyzer, Germany; Model-Vario EL III). Viscosity measurements of the polymer solutions were carried out with an Ubbelodhe viscometer (constant: 0.003899 ) at $25^{\circ} \mathrm{C}$. The viscosities were measured in aqueous solutions. The $\mathrm{pH}$ of the solution was neutral. The time of flow for solutions was measured at four different concentrations. From the time of flow of polymer solutions $(t)$ and that of the solvent $\left(t_{0}\right.$, for distilled water), relative viscosity $\left(\eta_{\text {rel }}=t / t_{0}\right)$ was obtained. Specific viscosity was calculated from the 
relation, $\eta_{\mathrm{sp}}=\eta_{\mathrm{rel}}-1$. Then, the reduced viscosity $\left(\eta_{\mathrm{sp}} / \mathrm{C}\right)$ and the inherent viscosity $\left(\ln \eta_{\text {rel }} / C\right)$ were calculated, where ' $\mathrm{C}$ ' is the polymer concentration in $\mathrm{g} / \mathrm{dl}$. The intrinsic viscosity was obtained from the point of intersection after extrapolation of two plots, i.e. $\eta_{\mathrm{sp}} / \mathrm{C}$ versus $\mathrm{C}$ and $\ln \eta_{\text {rel }} / C$ versus $C$, to zero concentration.

Thermo gravimetric analysis (TGA) of psyllium and that of the Psy-g-PMA were carried out with TGA instrument (Model: DTG-60; Shimadzu, Japan) from 25 to $750{ }^{\circ} \mathrm{C}$ keeping heating rate uniform in all cases at $5{ }^{\circ} \mathrm{C} / \mathrm{min}$. The differential scanning calorimeter analysis (DSC) of psyllium and that of Psy-g-PMA were carried out with DSC instrument (Model: Q 10 V9.4 Build 287 Universal). The study was performed in an inert atmosphere (nitrogen) from 20 to $270{ }^{\circ} \mathrm{C}$.

Scanning electron microscopy (SEM) studies were conducted by scanning electron microscopy (SEM) in powdered form (Model: JSM-6390LV, Jeol, Japan) to determine the morphological changes on the surface of both psyllium and Psy- $g$-PMA.

\section{Flocculation}

Flocculation studies were carried out by taking various grades of synthesized polymer (Psy-g-PMA) by standard Jar test procedure, in $0.25 \%$ kaolin suspension. All flocculation studies were done in jar test apparatus (makeIkon India). The test protocol involved taking a measured quantity of $800 \mathrm{ml}$ of the $0.25 \%$ suspension in $1,000 \mathrm{ml}$ Scott Duran glass beaker. Calculated amount of the flocculant psyllium and various grades of Psy- $g$-PMA were added in concentrated solution form to desired concentration.

The effectiveness of synthesized Psy-g-PMA as flocculant for wastewater treatment was carried out in three sets.

In the 1st set (set I control set), $800 \mathrm{ml}$ of the municipal sewage wastewater was stirred for $15 \mathrm{~min}$, at $60 \mathrm{rpm}$, then left to settle for $25 \mathrm{~min}$, at the end of which $25 \mathrm{ml}$ supernatant liquid was drawn from its top layer. The result of analysis from this control batch determines the inherent (natural) settling ability of the wastewater i.e. the results from this batch acts as a baseline with respect to which the flocculation efficacy of the grafted copolymers were determined.

The 2 nd set (set II) also consisted of $800 \mathrm{ml}$ of the same municipal sewage wastewater treated through the same procedure as above, but in presence of $2.5 \mathrm{ppm}$ of psyllium.

The 3rd set (set III) also consisted of $800 \mathrm{ml}$ of the same municipal sewage water treated through the same procedure as above, but in presence of 2.5 ppm of Psy-g-PMAIII (i.e. the optimized microwave grade as per our study).
The supernatant liquids drawn as described above, from the three sets of experiments were subjected to the following chemical/environmental analysis:

1. Turbidity testing using calibrated nephelo-turbudity meter.

2. Determination of total solid (TS), total dissolved solid (TDS), and total suspended solid (TSS); by gravimetric method (Greenberg, 1999).

\section{Result and discussion}

Structural characterization of psyllium and Psy- $g$ PMAIII

The intrinsic viscosity was evaluated for psyllium (intrinsic viscosity 2.55) and various grades of Psy-g-PMA, as shown in the Table 1. It is clear from the table that intrinsic viscosities of all the grades of Psy- $g$-PMA (best grade, Psy-g-PMAIII having intrinsic viscosity 6.93) are greater than that of psyllium (intrinsic viscosity 2.55). This can be explained by higher molecular weight of Psy- $g$-PMAIII than that of psyllium, due to the grafting of PMA on the main polymer backbone. This satisfy the Mark- Houwink- Skurada relation (intrinsic viscosity $\eta=\mathrm{KM}^{\alpha}$, where $\alpha$ and $\mathrm{K}$ are constants and both are related to the stiffness of the polymer chain. On the basis of percentage grafting (58\%) as given in Table 1, Psy- $g$-PMA Gr. III has been taken as the best synthesized grade.

The elemental analysis Psyllium and Psy- $g$-PMA III (the best grade of the grafted psyllium synthesized) are shown in Table 1. The estimation of five elements i.e., carbon, hydrogen, nitrogen, oxygen and sulphur was undertaken and the results have been summarized in Table 2. Increase in the percentage of carbon shows increased molecular weight due to grafting which was confirmed by intrinsic viscosity of grafted copolymer.

Figure 2 shows the FTIR spectrum of psyllium and Psy$g$-PMAIII. Psyllium mucilage showed several peaks and designation of each peaks are given below.

- 3,739.97 and 3,614.6 $\mathrm{cm}^{-1}$ : O-H stretching absorption of carbohydrate,

- 2,939.52 and $2,885.51 \mathrm{~cm}^{-1}: \mathrm{CH}_{2}$ asymmetric stretching,

Table 2 Elemental analysis results of psyllium and Psy-g-PMAIII

\begin{tabular}{llllll}
\hline Sample designation & \multicolumn{6}{c}{ Elemental compositions } \\
\cline { 2 - 6 } & $\mathrm{C}$ & $\mathrm{H}$ & $\mathrm{N}$ & $\mathrm{O}$ & $\mathrm{S}$ \\
\hline Psy & 41.11 & 6.351 & 0 & 52.5 & 0 \\
MA & 55.81 & 7.02 & 0 & 37.17 & 0 \\
Psy-g-PMAIII & 46.01 & 6.574 & 0 & 47.39 & 0 \\
\hline
\end{tabular}



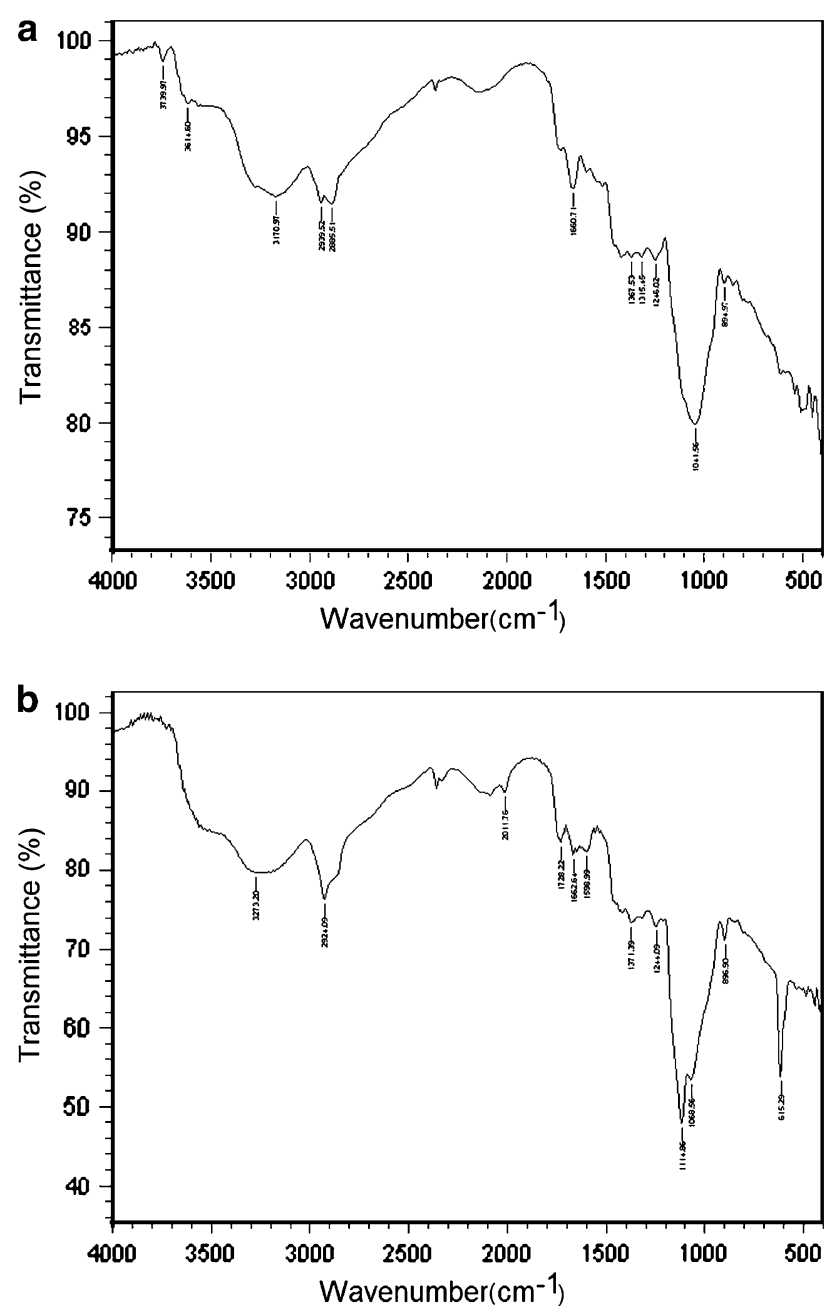

Fig. 2 FTIR spectra of a psyllium b Psy-g-PMA Gr. III

- $1,337.53,1,315.45$ and $1,245.02 \mathrm{~cm}^{-1}: \mathrm{CH}, \mathrm{CH}_{2}$ and $\mathrm{OH}$ in plane bending in carbohydrate,

- $1,014.56 \mathrm{~cm}^{-1}$ : $\mathrm{C}-\mathrm{O}$ stretching region as complex bonds, resulting from $\mathrm{C}-\mathrm{O}$ and $\mathrm{C}-\mathrm{O}-\mathrm{C}$ stretching vibrations,

- 894.97 and $545 \mathrm{~cm}^{-1}$ : pyranose ring.

Psy- $g$-PMAIII on the other hand, showed peaks at $1,662.64 \mathrm{~cm}^{-1} \quad(\mathrm{C}=\mathrm{O}$ stretching of $\mathrm{COOH}$ group $)$, $2,924.09 \mathrm{~cm}^{-1}\left(\mathrm{CH}_{2}\right.$ stretching $), 896.50 \mathrm{~cm}^{-1}\left(\mathrm{CH}_{2}\right.$ in plane bending) and $615.29 \mathrm{~cm}^{-1}(\mathrm{OCOH}$ defragmentation of $\mathrm{COOH}$ ), apart from the peaks obtained with Plantago psyllium mucilage. All the bands mentioned above are related to PMA and hence it is confirmed that grafting of PMA on psyllium has been carried out successfully.

Thermal study of psyllium and Psy- $g$-PMAIII

The TGA curves of psyllium (Fig. 3a) essentially involve two distinct zones of weight loss. The initial weight loss is $11.149 \%$ between temperatures $25-145^{\circ} \mathrm{C}$ which is due to the traces of moisture present. Again weight loss of $83.10 \%$ is observed up to $750{ }^{\circ} \mathrm{C}$ which is due to degradation of psyllium backbone. Psy-g-PMAIII (Fig. 3b) in addition to the above zones of weight loss, have two extra zones of weight loss $7.69 \%$ between $25-205{ }^{\circ} \mathrm{C}$ and $48.29 \%$ up to $750{ }^{\circ} \mathrm{C}$, due to the breakdown of backbone grafted psyllium and PMA chains.

The DSC curve of psyllium (Fig. 4) gives broad endothermic peak at $80.89{ }^{\circ} \mathrm{C}$ (temp range 34.44 to $138{ }^{\circ} \mathrm{C}$ ) due to the traces of water molecule. The broad endothermic peak in Psy-g-PMAIII slightly increased from 80.89 to $82.50{ }^{\circ} \mathrm{C}$. In addition, two other peaks one endothermic at $218.50{ }^{\circ} \mathrm{C}$ and one exothermic peak at $220.83{ }^{\circ} \mathrm{C}$ was also observed. The generation of additional peaks confirms grafting (Fig. 4) of PMA on psyllium backbone.

Morphological study of psyllium and Psy-g-PMAIII

Figure 5a and $\mathrm{b}$ show the cross-section morphologies of native and grafted psyllium. Both the Figures clearly show the marked differences. Homogeneous surface was observed for psyllium but Psy-g-PMAIII showed heterogeneous network structure. Similar types of results were reported by authors (Mishra et al. 2002a, 2004).
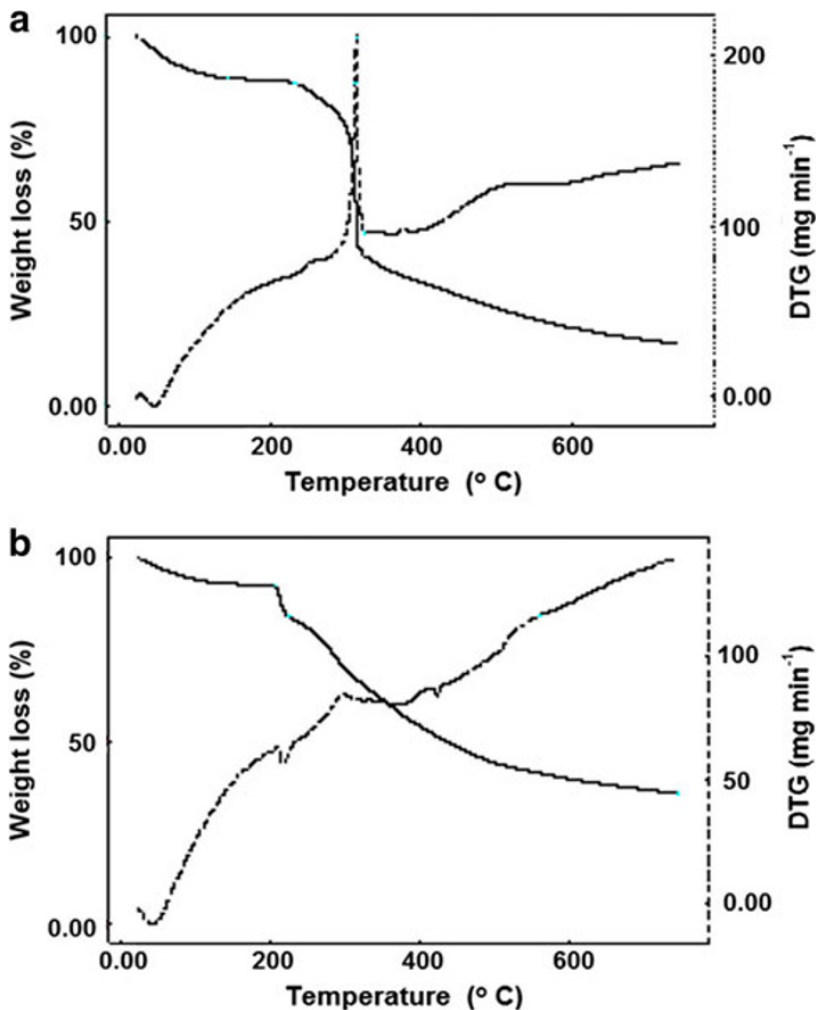

Fig. 3 TGA (line) and DTG (dashed line) curves of a psyllium b Psy-g-PMA Gr. III 


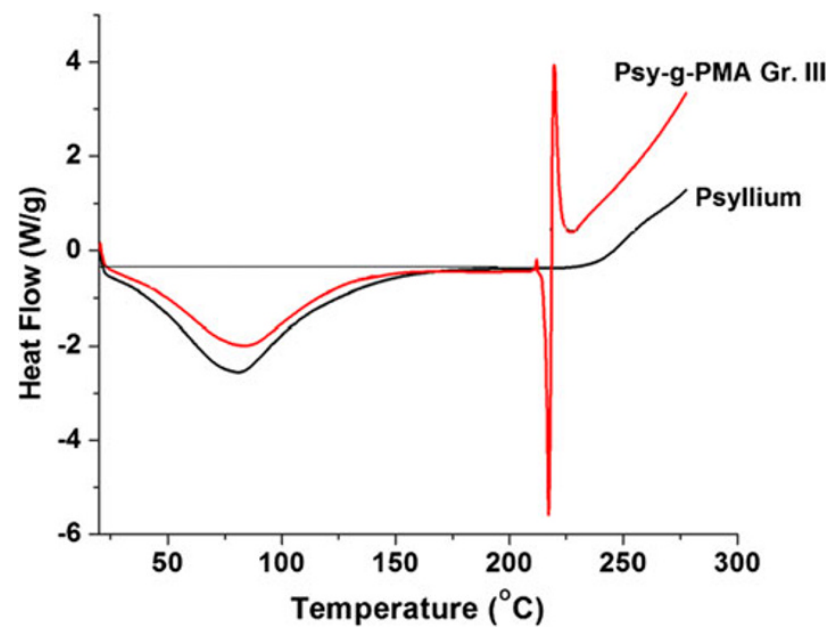

Fig. 4 DSC curves of a psyllium b Psy- $g$-PMA Gr. III
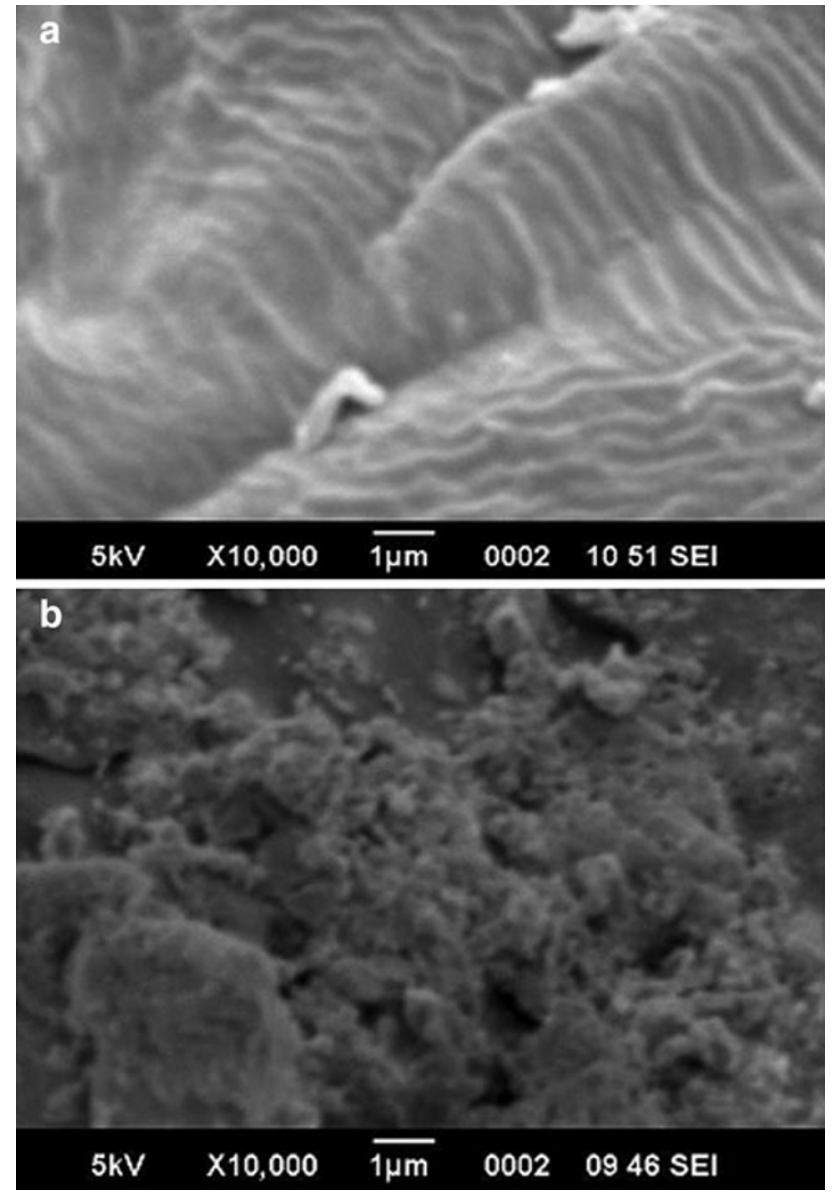

Fig. 5 SEM micrographs of a psyllium and b Psy-g-PMA Gr. III

Application of psyllium and Psy-g-PMAIII as flocculant

Table 3 shows the values of different parameters of wastewater treated with natural psyllium and Psy-g-
Table 3 Flocculation characteristics of psyllium and Psy-g-PMAIII

\begin{tabular}{llll}
\hline Parameter & $\begin{array}{l}\text { Supernatant } \\
\text { liquid from Set I } \\
\text { (i.e. waste water } \\
\text { without } \\
\text { flocculant) }\end{array}$ & $\begin{array}{l}\text { Supernatant } \\
\text { liquid from Set II } \\
\text { (i.e. waste water } \\
\text { with 2.5 ppm P. } \\
\text { psyllium) }\end{array}$ & $\begin{array}{l}\text { Supernatant liquid } \\
\text { from Set III (i.e. } \\
\text { waste water with } \\
\text { 2.5 ppm Psy-g- } \\
\text { PMAIII) }\end{array}$ \\
\hline Turbidity & 37 & 27 & 12 \\
TS (ppm) & 408 & 300 & 226 \\
TDS & 291 & 243 & 212 \\
(ppm) & & & \\
$\begin{array}{c}\text { TSS } \\
\text { (ppm) }\end{array}$ & 117 & 57 & 14 \\
\hline
\end{tabular}

PMAIII. The results of this analysis are important for determination of applicability of Psy- $g$-PMA as a flocculant for treatment of municipal wastewater. Wastewater shows high concentration of turbidity, total solids (TS), total dissolved solids (TDS), total suspended solids (TSS). 2.5 ppm of Psy- $g$-PMAIII gives better result and maximum turbidity removal from 100 to 67 NTU. There is decrease in concentration in all parameters with the introduction of psyllium and Psy- $g$-PMAIII. It is important to note that Psy- $g$-PMA III resulted in higher decrease in the parameters compared to psyllium. Hence the result shows the possible application of grafted psyllium in wastewater treatment.

\section{Conclusion}

The overall performance of optimized grade of both grafted copolymers and psyllium as flocculant for waste water treatment has been investigated. As the percentage of grafting is higher in grafted copolymer, it shows higher intrinsic viscosity from (3.47 to 6.93). Optimized best grade i.e., Psy- $g$-PMAIII shows higher flocculation efficacy of grafted copolymer which reduces turbidity, TS, TDS, and TSS in appreciable amount as compared to psyllium. Better flocculation efficacies of grafted copolymers are due to their structural differences. Here psyllium has linear structure where as grafted psyllium have web like structure due to polymethacrylic acid chain grafted onto rigid psyllium backbone. All the experimental results reported in this paper shows better flocculation efficacies due to higher molecular weight indicating longer chain length of grafted PMA; i.e. higher radius of gyration of the macromolecules.

Acknowledgments The authors gratefully acknowledge the generous and kind cooperation of Professor (Dr.) Mrs. Usha Jha, Head, Department of Applied Chemistry, Birla Institute of Technology, Mesra, Ranchi, India to carry out this work. 
Open Access This article is distributed under the terms of the Creative Commons Attribution License which permits any use, distribution, and reproduction in any medium, provided the original author(s) and the source are credited.

\section{References}

Agarwal M, Rajani S, Mishra A (2001) Study of flocculation efficiency of Okara gum in sewage wastewater. Macromol Mater Eng 286:560-563

Bolto BA, Dixon DR, Gray SR, Ha C, Harbour PJ, Ngoc L, Ware AJ (1996) The use of soluble organic polymers in waste treatment. Water Sci Technol 34(9):117-124

da Silva DA, de Paula RCM, Feitosa JPA (2007) Graft copolymerization of Acrylamide onto cashew gum. Eur Polym J 43: 2620-2629

Gupta KC, Sahoo S (2001) Graft copolymerization of acrylonitrile and methacrylate comonomers on cellulose using ceric ions. Bio Macromol 2:239-247

Kang DW, Choi RC, Kweon DK (1999) Stability constant of Amidoximaed chitosan-g-Poly(Acrylamide) copolymer for heavy metal ions. J Appl Polym Sci 73:469-476

Khalil MI, Farag S (1998) Utilization of some starch derivative in heavy metal ions removal. J Appl Polym Sci 69:45-50

Mishra A, Rajani S, Agarwal M, Dubey R (2002a) P. Psyllium-gPolyacrylamide: synthesis and characterization. Polym Bull 48:439-444

Mishra A, Rajani S, Dube R (2002b) Flocculation of textile wastewater by plantago Psyllium mucilage. Macromol Mater Eng 287:592-596

Mishra A, Yadav A, Agarwal M, Rajani S (2004) Polyacrylonitrile grafted Plantago psyllium mucilage for the removal of suspended and dissolved solid from tannery effluent. Colloid Polym Sci 282:300-303

Mishra S, Mukul A, Sen G, Jha U (2011) Microwave assisted synthesis of polyacrylamide graft starch (St-g-PAM) and its applicability as flocculant for water treatment. Intern $\mathrm{J}$ Biol Macromol 48:106-111

Mostafa KM (1995) Graft polymerization of acrylic acid onto starch using potassium permanganate acid (redox system). J Appl Polym Sci 56:63-269

Rajani S, Agarwal M, Mishra A (2002) Synthesis of Plantago psyllium mucilage grafted and its flocculation efficiency in textile effluent water. Qual Res J Canada 37:371-378

Samantaroy S, Mohanty AK, Misra M (1997) Removal of hexavalent chromium by Kendu fruit gum dust. J Appl Polym Sci 66: $1485-1494$

Sen G, Pal S (2009) Polyacrylamide grafted carboxymethyl tamrind (CMT-g-PAM): development and application of a novel polymeric flocculant. Macromol Symp 277:100-111

Sen G, Kumar R, Ghosh S, Pal S (2009) A novel polymeric flocculant based on polyacrylamide grafted carboxymethylstarch. Carbohydrate Polymer 77:822-831

Shin KS, Choi EM, Hwang TS (2011) Synthesis, and characterization of cation-exchange membrane based on sulfonated Sty/HEA/ LMA terpolymer. J Appl Polym Sci 119:3180-3188

Singh RP, Karmakar GP, Rath SK, Pandey SR, Tripathy T, Panda J, Kanan K, Jain SK, Lan NT (2000) Novel biodegradable flocculants based on polysaccharides. Curr Sci 78:798-803

Singh RP, Karmaker GP, Rath SK, Pandey SR, Tripathy T, Panda J, Kanan K, Jain SK, Lan NT (2002) Biodegradable brag reducing agents and flocculants based on polysaccharides. Polymer Engg Sci.x 40:46-60

Udaybhaskar P, Iyengar L, Prabhakara RAVS (1990) Removal of heavy metal from industrial wastewater using chitosan coated oil palm shell charcoal. J Appl Polymer Sci 39:739-747

Vahdat A, Bahrami H, Ansari N, Ziaie F (2007) Radiation grafting of Styrene onto polypropelene fibers by $10 \mathrm{MeV}$ electron beam. Radiat Phys Chem 76:787-793

Wang JP, Chen YZ, Zhang SJ, Yu HQ (2008) A Chitosan based flocculant prepared with gamma-irradiation induced grafting. Biores Technol 99:3397-3402 